\title{
Energy Saving Mechanism for a Smart Wearable System: Monitoring Infants during the Sleep
}

\author{
Duarte Fernandes ${ }^{1}$, André G. Ferreira ${ }^{1}$, Sérgio Branco ${ }^{1}$, Reza Abrishambaf ${ }^{1,2}$, Hélder Carvalho ${ }^{3}$, José Mendes $^{1}$, Jorge Cabral ${ }^{1}$, \\ Ana Rocha ${ }^{3}$ \\ Centro Algoritmi, University of Minho, Campus of Azurém, 4800-058, Guimarães, Portugal ${ }^{1}$ \\ Department of Engineering Technology, Miami University, Hamilton, OH, 45011, USA ${ }^{2}$ \\ Center of Textile Science and Technology (2C2T), University of Minho ${ }^{3}$
}

\begin{abstract}
In Smart Wearable Systems (SWS), the wearable devices are powered by batteries with very limited energy available. These emergent systems have strong Quality of Service $(\mathrm{QoS})$ requirements, with focus on reliable communication and low power consumption. This is the scope of the Baby Night Watch, a project developed in the context of the European Texas Instruments Innovation Challenge (TIIC) 2015. This Project consists of a monitoring tool for infants, which matches different emergent research fields. SWSs require energy saving mechanism to reduce the energy wasting during wireless communications. A Transmission Power Control (TPC) mechanism that changes its characteristics according to the scenario of operation, is proposed. It uses sensors to determine the position of the infant and, based on that, predicts the current state of the channel. Other TPC algorithms are implemented and their performance are compared with our novel mechanism. The proposed TPC mechanism outperforms the existing ones in terms of the energy saving.
\end{abstract}

Keywords - Transmisison Power Control (TPC) Mechanisms; Smart Wearable Systems (SWS); Wearable Devices;

\section{CENTRO INTRODUCTION}

Nowadays, the recent developments on wireless communication and device miniaturization have led to the development of portable devices, and more recently wearable devices. Due to its nature, the Smart Wearable Systems (SWS) have potential to be integrated in several applications. according to [1], [2], the main requirements of the SWS are: 1) reliable communication: the system has to ensure a reliable connection among devices, i.e. a packet error rate (PER) lower than $10 \%$; 2) low latency: for medical applications the maximum delay allowed on communications is $150 \mathrm{~ms}$, and in non-medical applications is $250 \mathrm{~ms}$; and 3) energy-efficient: depending on the application it must have the ability to operate for long periods of time (hours or even days) without any intervention. In SWS, the data packets must be delivered efficiently and, as the devices are powered by small batteries, the minimum energy needed must be used to ensure it. The main source of energy consumption in Wireless Sensor Network (WSN) and Wireless Body Sensor Network (WBSN) is the communications, near of $60 \%$ of the total consumption of the system [2]. In this context, the use of strategies like Transmission Power Control mechanisms (TPC) have drawn, recently, special attention by the scientific community. Smith et al. states that TPC mechanisms must be an integral part in future developments of wireless networks operating near human body [3]. However, unlike traditional wireless networks, these mechanisms developed for SWS have to deal with the highly dynamic channel quality. The movement of the human body and the multi-path components - due to reflecting objects in the surrounding environment - result in significantly path-loss (higher than $40 \mathrm{dBm}$ ) and fading [4].

The Output Power Transmission Level (OPTL) used by the sensor nodes in SWS can be pre-defined or dynamically configured and updated. In literature we can notice that in SWS the authors use a pre-defined OPTL, usually the maximum available, to ensure the requirement of reliable communication. However, this is a wrong trendy and should be avoided. It increases, significantly, the energy consumption, the RF interference among systems or coexistent devices and heat dissipation [5], [6]. In dynamic OPTL management, the idea consists in the use of the lowest OPTL necessary to ensure the correct delivery of the data packet at the receiver [7]. This is the principle of the TPC mechanism, which explores the features of the radio devices (they offer several OPTL) changing the OPTL dynamically, according to the channel condition [8]. TPC mechanisms aims to extend the life time of the sensor nodes while ensure the remains requirements. A precise comprehension of the channel behavior - through an exhaustive experimental characterization of the channel - in several scenarios, allow us to find and adapt, suitable features of others TPC mechanisms for the SWS. Therefore, a closedloop architecture is proposed based on RSSI considered as main metric in current channel state determination. Besides that, the proposed algorithm makes use of the sensors, already present in the SWS, to understand the magnitude of dynamism channel behavior expected. This information is extremely important, as it allows the system to select the adequate control based on channel state prediction.

The rest of the paper is organized as follows. In Section II the related works is discussed. Section III describes the architecture of the Baby Night Watch and the corresponding devices. In section IV, preliminary studies are presented. In Section V, the proposed algorithm is discussed in detail. Section VI, presents the experimental results and, finally, Section VII concludes the paper. 


\section{RELATED WORK}

TPC mechanism operation flux consists of two processes: (1) interpretation of the current channel state using a metric or set of metrics, and (2) OPTL mechanism of control.

The perception of the current channel state can be made through a feedback closed-loop architecture or using a Link Quality Estimator (LQE) [9]. In TPC mechanisms with a closed-loop architecture, the OPTL - configured by the sensor node to transmit the data packets - is determined by the sink node. Most of the TPC algorithms proposed by literature are based on this approach, e.g.[6], [8], [10], [11]. These methods have been demonstrated good improvements in terms of energy saving. Energy savings can be near of $35 \%$ [10] for dynamic movements and, $51.63 \%$ for less dynamic movements [11]. These mechanisms can opt only for a single metric (RSSI) [6], [8], [10] or combination of metrics (RSSI and LQI) [11]. However, the number of feedback packets emitted by the sink node is significant (with averages of 11 frames for every 100 packets received), especially in dynamic scenarios. Some solutions were proposed in the literatures to reduce the number of feedback packets. Quwaider et al. introduced for the first time the RSSI range target [6], however the number of control packets is still significant. In [3] and [12] TPC mechanisms with a variable adaptive feedback were introduced, but when the interval time among control packets increases, the performance of the metrics reliability and energy consumption decreases. TPC mechanisms based on LQE use accelerometers attached in the body of the user to detect the posture and movement, to posterior predict channel state. In [6], the authors determined the point, when user walking, that there is a high chance of having a better link (chest-wrist) quality. When reached this point, the sensor transmits the data packet with a reduced OPTL, saving $35.6 \%$ of the total energy consumption but the latency is increased. Other works based on LQE, include a higher number of input variables of the LQE model. Vallejo et al. considered for the first time the impact of body characteristics (body fat, upper arm circumference, lower arm length) and body position [7]. This LQE is based on the artificial neural network and Fuzzy logic, reaching a good RSSI estimation for different postures (Root-Mean-Square Error $<7 \%$ ). Similar work was proposed in proposed in [13], where the LQE uses the a Kalman filter to predict the current RSSI, based on the accelerometer to interpret user posture. The TPC mechanism based on LQE removes the need of control packet but, requires an exhaustive and accurate channel characterization. In all the mentioned scenarios, they have been limited to static scenarios; no movement as well the fading has been considered. In the context of OPTL control, two widely used algorithms are: 1) linear/conservative: it changes the OPTL gradually according to the current channel state; 2) binary or dynamic (so-called aggressive approach): the OPTL is changed exponentially [8].

TPC mechanisms developed for WSN are not suitable for SWS, because they are designed for wireless systems with high density of nodes, with reduced, or even without any dynamism. The TPC in WBSNs have been designed for the links that composed on-body communication where the distance among devices may vary in every communication.

\section{SYSTEM DESIGN AND ARCHITECTURE}

\section{A. System Overview}

Fig. 1 illustrates the architecture of the Baby Night Watch. The monitoring subsystem is composed of sensors and a sink node, so-called Gateway, which has the responsibility for managing the network. In order to implement a long term continuous monitoring system, the sensor node, called Wearable IoT Device, is sufficiently small, light and comfortable.

The sensor node collects different types of physiological data and sends those parameters to the Gateway (inside the communication range of the chest belt). This off-body communication is made using the IEEE 802.15.4-compilant wireless transceiver radio, SoC CC2530, over the $2.4 \mathrm{GHz}$ Industrial Scientific and Medical (ISM) band. The Gateway, beyond the ability to communicate with the Wearable IoT Device, is able to transmit the received data to others devices, located outside of the ZigBee Coordinator Range, through WiFi. This makes the information available for real-time query. A detailed description of the SWS and its devices is presented in [14].

\section{B. Wearable IoT Device}

The device responsible for the acquisition of physiological parameters of the infant is designed using Wearable IoT Device, and it is illustrated in Fig. 2. After the acquisition and parsing of the parameters, the device sends the processed data to the Gateway, using the CC2530 SoC from Texas Instruments, through ZigBee wireless technology (Z-Stack API).

This device consists in a PCB with reduced dimensions $(4.3 \times 3.8 \times 0.9 \mathrm{~cm})$ as a way to respect the social requirements of wearable devices. In this device, a set of sensors such as: heart rate sensor which is composed of the instrumentation of the sensor and the textile electrodes integrated on the wearable chest band; position sensor, which consists of an accelerometer for the identification of the different positions; breathing sensor, which measures the breathing rate using to the same accelerometer used in the position sensor; and body temperature sensor, which measure the temperature of the

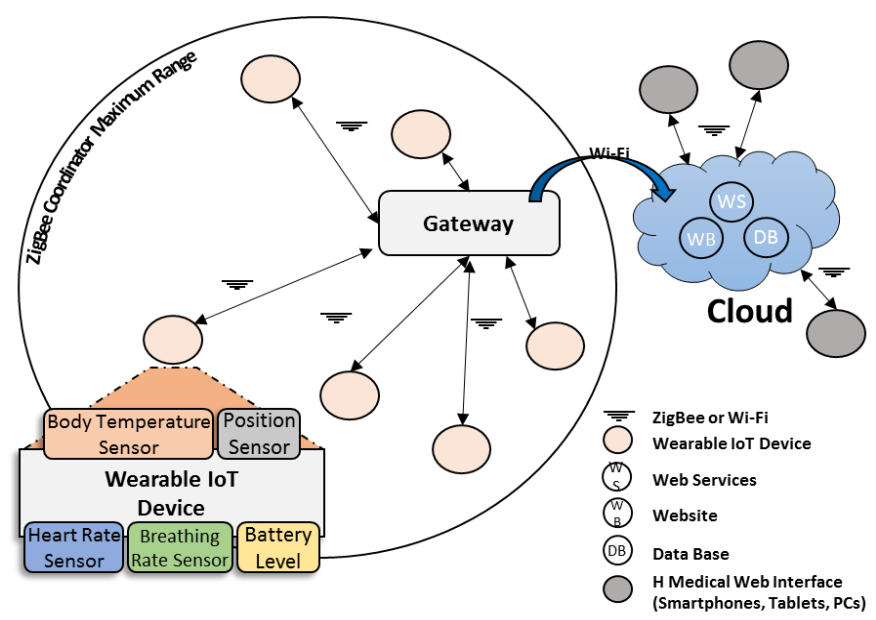

Fig. 1. Baby Night Watch's Overview [14]. 


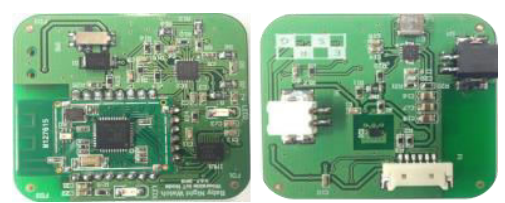

Fig. 2. PCB implementation of the Wearable IoT Device (Top and Bottom).

infant through an infrared thermopile sensor. A summary of the electronic components that constitute the wearable IoT Device is showed in TABLE I.

The CC2530 is an IEEE 802.15.4-Compliant wireless transceiver radio from Texas Instruments that operates in the 2.4GHz Industrial Scientific and Medical (ISM) band. The possible output powers of the selected SoC goes from $-22 \mathrm{dBm}$ up to $4.5 \mathrm{dBm}$. The difference of energy consumption between the higher and lower levels is higher than the total energy consumption of the MCU $(<10 \mathrm{~mA})$. Thus, the exploration of a proper OPTL management appears, naturally, as a potential efficient solution to reduce the overall energy consumption.

\section{ENERGY SAVING MECHANISM}

Low power consumption is a critical characteristic in the Baby Night Watch project, which has to operate for hours, preferably more than 8 hours. Main source of energy consumption in the Wearable IoT Device is the wireless communication (68\%). The remaining energy consumption is divided by the processing subsystem $(12 \%)$ and the sensing process $(10 \%)$. Thus, the exploration of a proper OPTL management (TPC mechanisms) appears, naturally as a potential efficient solution to reduce the overall energy consumption of the systems.

\section{A. WBSN RF Off-Body Communications Features}

Two preliminary experimental tests were conducted, aiming to demonstrate the effect of the infant posture, surrounding environment and OPTL on the channel quality. Both experimental test were performed in an ordinary room (characterized for being a potential wave reflector environment), and the behavior of the off-body link was analyzed during the time. During this period of time, the user

TABLE I. Electrical Characteristics AND DATA RATE PRODUCED BY SENSORS

\begin{tabular}{|c|c|c|c|c|c|}
\hline \multirow{2}{*}{ Hw } & \multicolumn{5}{|c|}{ Hardware Data } \\
\cline { 2 - 6 } & Reference & $\begin{array}{c}\text { Op.Current } \\
(\boldsymbol{m A})\end{array}$ & $\begin{array}{c}\text { Resolution } \\
(\text { bits })\end{array}$ & $\begin{array}{c}\text { Sampling } \\
\text { Rate } \\
(\boldsymbol{H z})\end{array}$ & $\begin{array}{c}\text { Data } \\
\text { Rate } \\
(\text { bps })\end{array}$ \\
\hline MCU & $\begin{array}{c}\text { SoC-8051 } \\
\text { CPU Core }\end{array}$ & 6.5 & -- & -- & -- \\
\hline Transceiver & $\begin{array}{c}\text { SoC-RF } \\
\text { Transceiver }\end{array}$ & $\approx 30$ & -- & -- & -- \\
\hline Heart Rate & AD8232 & 0.2 & 24 & 0.07 & 2 \\
\hline $\begin{array}{c}\text { Position } \\
\text { Sensor }\end{array}$ & LSM330DLC & 8 & 24 & 0.96 & 0.65 \\
\hline $\begin{array}{c}\text { Breathing } \\
\text { Sensor }\end{array}$ & LSM330DLC & 8 & 24 & 0.07 & 2 \\
\hline $\begin{array}{c}\text { Body } \\
\text { Temp. }\end{array}$ & TMP007 & 10 & 16 & 0.05 & 0.8 \\
\hline $\begin{array}{c}\text { Tension } \\
\text { Level }\end{array}$ & ADC & -- & 24 & 0.02 & 0.5 \\
\hline
\end{tabular}

was invited to follow a predefined sequence of postures, namely: A (infant is lying on his backs); followed by B (lying on his side, with LOS to sink); C (lying on his side, opposite side of the sink); and finally posture D (infant lying on his stomach); while the data packets were emitted by sensor node to sink in a fixed OPTL $(0 \mathrm{dBm})$. Fig. 3 illustrates the effect of each position of the infant in the channel behavior. At most favorable off-body communications scenarios (scenario A and B), the RSSI is, essentially, due to Line-of-Sight (LOS) communication existence. The RSSI revealed satisfactory values and a behavior almost static, with short variations among samples, from 2 up to $4 \mathrm{dBm}$. The remaining scenarios, $\mathrm{C}$ and $\mathrm{D}$, are the worst for the wireless communications. The oscillation phenomenon can be explained by the fact the final RSSI is the combination of several multi-path components contribute, due the absence of the LOS component.

Thus, the effect of the surrounding environment is also present, and results in dynamic behavior of the received signal, i.e. extremely dynamic channel behavior in short periods of time (or among samples) and poor channel quality (RSSI values closer of the maximum receiver sensibility, $-92 \mathrm{dBm}$ in CC2530). The features of the electromagnetic waves, described above, suggest the inclusion of a target RSSI margin. In the case of RSSI be inside of the target margin, the OPTL will not be updated, otherwise a new value is computed.

Fig. 4 shows the box plot of the average RSSI obtained on the second preliminary experimental characterization of the link. This link was summited to different OPTL for every scenario considered. As depicted in the graphs, and as mentioned before, the most favourable scenarios to the wireless communication are scenarios A and B. This happens because, independently of the OPTL used, the average RSSI is always higher than the CC2531 receiver sensitivity, and as we can observe in TABLE II, there is no packet loss (PER of 0\%) and a low latency is achieved. However, it is necessary to refer that the two nodes are located at short distances, therefore, it is expected a reduction of performance in terms of average RSSI and PER when the distance among nodes increases.

For the scenarios C and D, we can observe that the RSSI is very close of the receiver sensitive when low OPTL are used. As a consequence, the percentage of packets lost and latency increase significantly. It is especially critical in scenario D. In this situation independently of the power transmission level selected, the average RSSI is very close to the receiver's minimum value. In those cases, it is possible to observe that the requirement of reliable communication is no longer respected,

Received Power Signal (OPTL de $0 \mathrm{dBm}$ )

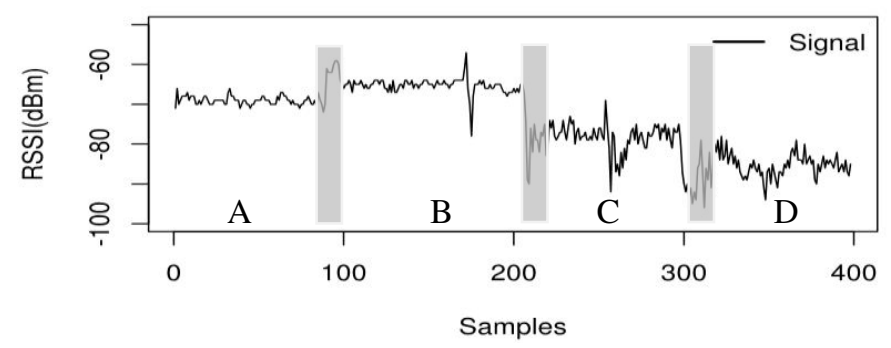

Fig. 3. Received power signal for different baby positions and the transitions among positions (identified by a grey rectangle). 

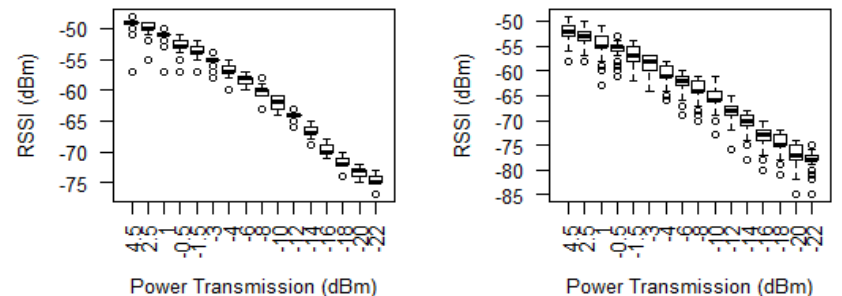

Channel Behaviour - Position C
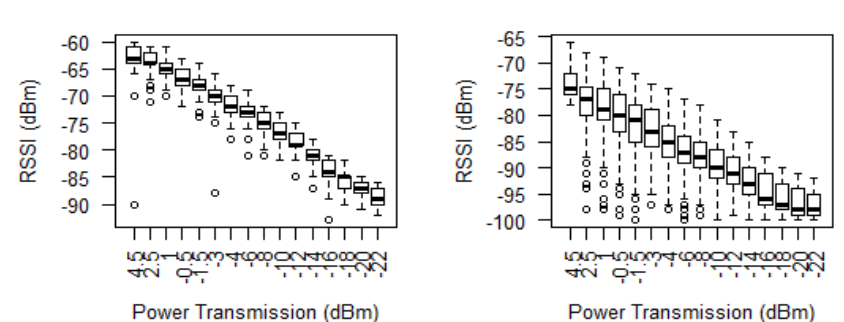

Fig. 4. Box plot of experimental average RSSI for the positions considered to the application.

TABLE II. RADIO PERFORMANCE IN TERMS OF THE METRICS PER LATENCY AND AVERAGE RSSI, IN DIFFERENT SCENARIOS AND PTX

\begin{tabular}{|c|c|c|c|}
\hline \multirow[b]{2}{*}{ Scenario } & \multicolumn{3}{|c|}{ Radio Performance } \\
\hline & Average RSSI (dBm) & $\operatorname{PER}(\%)$ & $\begin{array}{c}\text { Latency } \\
(\mathrm{ms})\end{array}$ \\
\hline A & $\begin{array}{l}-48.9(4.5 \mathrm{dBm}) ;-53 \quad(-0.5 \\
\mathrm{dBm}) ;-61(-8 \mathrm{dBm}) ;-73.2(- \\
20 \mathrm{dBm})\end{array}$ & $0 ; 0 ; 0 ; 0$ & $0 ; 0 ; 0 ; 0$ \\
\hline B & $\begin{array}{l}-52.1(4.5 \mathrm{dBm}) ;-55.6(-0.5 \\
\mathrm{dBm}) ;-63.8(-8 \mathrm{dBm}) ;-76.8 \\
(-20 \mathrm{dBm})\end{array}$ & $0 ; 0 ; 0 ; 0$ & $0 ; 0 ; 0 ; 0$ \\
\hline $\mathrm{C}$ & $\begin{array}{l}-63.7(4.5 \mathrm{dBm}) ;-66.5(-0.5 \\
\mathrm{dBm}) ;-74.6(-8 \mathrm{dBm}) ;-87.5(- \\
20 \mathrm{dBm})\end{array}$ & $\begin{array}{c}0 ; 0 ; 0.5 \\
1\end{array}$ & $\begin{array}{c}0 ; 0 ; 50 \\
50\end{array}$ \\
\hline $\mathrm{D}$ & $\begin{array}{llll}-73 & (4.5 \mathrm{dBm}) ; & -80.4 & (-0.5 \\
\mathrm{dBm}) ; & -87.7 & (-8 \mathrm{dBm}) ; & -96.7 \\
(-20 \mathrm{dBm}) & & \end{array}$ & $\begin{array}{c}0 ; 0.5 ; 11 ; \\
34\end{array}$ & $\begin{array}{c}0 ; 50 ; \\
150 ; 2400\end{array}$ \\
\hline
\end{tabular}

as there is a large percentage of packet losses and latency. For the scenario $\mathrm{C}$ we have the same phenomena for OPTL lower than $-18 \mathrm{dBm}$. This last preliminary analyses demonstrates why some scenarios requires a higher OPTL to achieve the desired requirements.

However, it also reveals that the power transmission mechanisms must lead with trade-offs among parameters. For instance, in static scenarios (as A and B) the use of a predefined OPTL means a waste of energy, because lowers OPTL are able to ensure a reliable communication $(\mathrm{PER}=0 \%)$, and at same time increases the energy efficiency of the system. However, in dynamic scenarios the use of lowers OPTL can save energy but, at same time, will be negatively reflected this on latency and reliable communication (the number of packet losses will increase). The trade-off between the power consumption and the other parameters is usually guaranteed through the use of a target RSSI range.

\section{B. Wireless RF Off-Body Preliminary Tests}

As mentioned in state of art of TPC, these mechanisms can follow one of the following approaches: linear TPC, binary TPC, or a dynamic TPC (combination of the two firsts). It has been proved that the design of the TPC to be implemented must consider two different channel behaviors.

Through the previous preliminary experimental tests, two distinct channel environments were identified, a small-signal and wide-signal variations environment. For this reason, it is expected that the final TPC mechanism implemented for the present application follows a dynamic approach, being able of following the dynamism of the environment.

Our approach consists of the combination of the metric RSSI and the information available through potential sensors distributed over the body. In this specific application we intend to use the information of an accelerometer. Two techniques of OPTL determination are used in the Baby Night Watch system, a conservative and an aggressive TPC mechanism. Preliminary tests were performed, aiming to introduce a new vision about working principle, limitations of the two TPC techniques, and finally, a comparative analysis between them. During practical tests, four user states/positions were considered. Tests were performed in an indoor environment (sleeping room $4.0 \times 3.0$ $\mathrm{m})$, the Wearable IoT Device was located on the chest belt of the user and the Gateway was located bedside the table (distance among devices $\approx 3 \mathrm{~m}$ ). The selected target RSSI range is from $-87 \mathrm{dBm}$ up to $-80 \mathrm{dBm}$, and the data from the sensor node is collected every 3 seconds, during a period of 1.5 minutes for every user state. Fig. 5 (left), depicts the behavior of the signal over time in a static scenario (position B), while the Baby Night Watch apply the conservative and aggressive TPL mechanism. Through the comparison of both methods, it is possible to verify that the aggressive mechanism is faster than the conservative, as the latter requires more than eight control frames to reach the Optimal Minimum Output Power (OOP), i.e. the minimum output power to guarantee a successful delivery of packages, which was $-14 \mathrm{dBm}$ in present experimental test, against the 2 frames of the aggressive mechanism. Fig. 5 (right), demonstrates that in a dynamic environment, like the user in position $\mathrm{C}$ and $\mathrm{D}$, the signal does not stay within the target RSSI range for long periods at the same OPTL (channel conditions vary rapidly). In scenario $\mathrm{C}$,
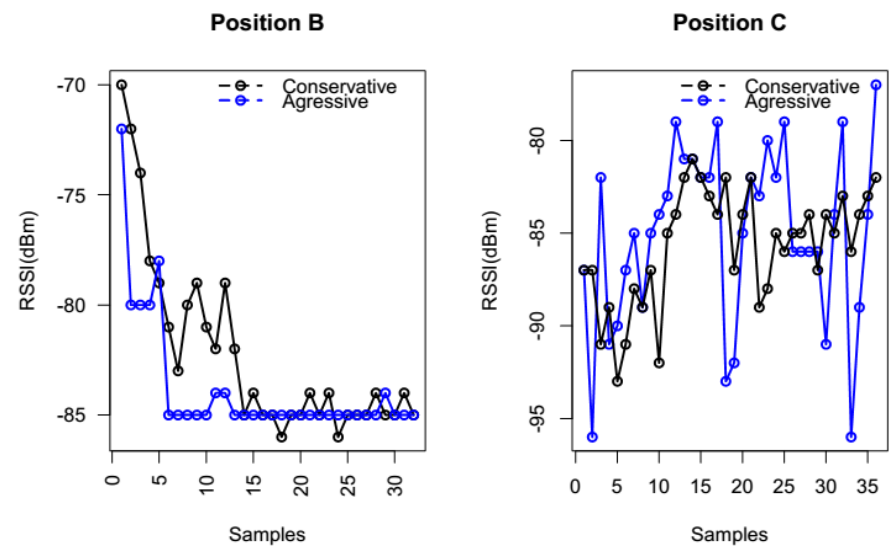

Fig. 5. Wearable IoT signals as result of application of Aggressive and Conservative TPL in a static and dynamic environment, respectively position $\mathrm{B}$ and $\mathrm{C}$. 
the aggressive mechanism adjusts the OPTL exponentially, as a consequence the current goes farther from the target RSSI margin (switched 14 control frames until reaching to the OOP), while the conservative adjusts the OPTL linearly around the target RSSI range (switched 6 control frames).

As a result, the aggressive TPL is the most suitable for static environment, while in dynamic environments, the conservative needs less control frames to reach the OOP and to keep within the target RSSI range for longer periods of time.

\section{PROPOSED DYNAMIC TPC FOR INFANTS SLEEPING}

The proposed algorithm adjusts the OPTL based on the user position - it is run time determined by Wearable IoT Device, which is periodically transmitted to the Gateway, through the algorithm presented and validated in [14] - and on current state of the channel. Two OPTL control mechanisms are considered and the commutation between them is selected based on the infant position information, obtained through the accelerometer. A detailed description of the algorithm designed to detect the position of the infant and its experimental validation is presented in [14]. The conservative mechanism changes the OPTL linearly for dynamic environments, while the aggressive mechanism changes the OPTL exponentially. This approach seems suitable for dynamic scenarios, aiming to reduce the number of control frames sent to the Wearable IoT Device until it reaches the OOP, while following the dynamic behavior of the channel.

\section{A. Pseudocode}

The proposed TPC mechanism is based on closed-loop architecture. The sensor node starts the communication with the sink node, sending the data packets with the maximum output power allowed in by IEEE 802.15.6 standard $(0 \mathrm{dBm})$ [15]. Consequently, the sink node determines the current channel state based on the RSSI of the data packet, or set of packets received. Posteriorly, the sink node is responsible for determining the OOP. Information about the new OOP is send to the sensor node in form of control frame at maximum OPTL. The sensor node will upgrade the OPTL, every time it receives a control frame. Future data frames are sent to the sink with the new OPTL. This process is repeated during the sensor nodes' lifecycle.

The pseudocode of the proposed TPC algorithm, described before, is shown in Pseudocode 1, which includes the two scenarios expected for the channel behavior during the operation of the SWS. The pseudocode of the algorithm starts getting the previous OPTL, i.e. the power transmission used by the sensor node to transmit the current received data packet. The RSSI relatively to the packet received is obtained, as well as the current position of the infant. These information are the input data of the algorithm. Based on the scenario, the algorithm selects the conservative or aggressive mechanism. When selected, the algorithm checks if the RSSI of the received packet is within or outside of the target RSSI range. When it is within the range, the algorithm does not proceed any change, and the process end until a new data packet received. If the current RSSI value is higher than the maximum value of the target RSSI range, the OPTL will be reduced to avoid

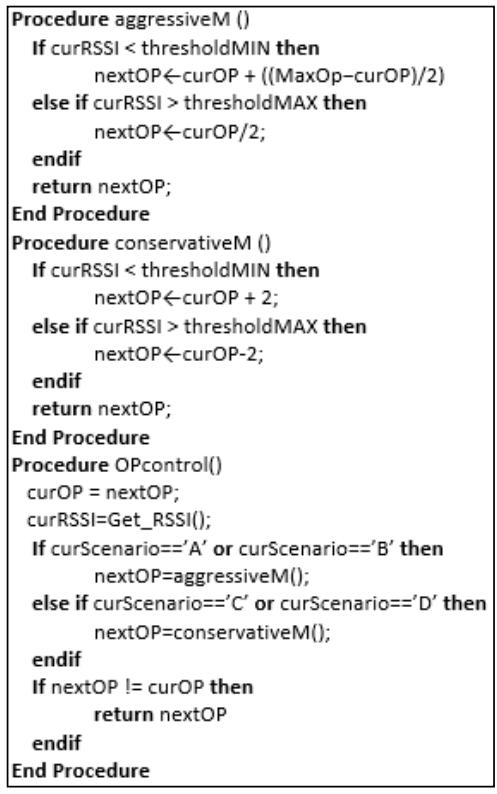

Pseudocode 1. Pseudocode of the proposed TPC mechanism.

unnecessary energy consumption. If the current RSSI value is lower than the minimum RSSI of the range, the OPTL will be increased based on the respective control mechanism, in order to prevent losses of packets or increase the latency of the communication.

\section{RESULTS}

Experimental evaluation tests were conducted, following the same procedure of the preliminary tests. The evolution of TPC signal and the signal emitted with an OPTL of $0 \mathrm{dBm}$ is graphically represented in Fig. 6. It is easily noticeable that the TPC algorithm revealed to be able to follow the dynamism of the channel communication environment. This is highlighted at the transition between positions (represented by the gray bars), where there is a fast-acting mechanism, ensuring that the signal remains within the predefined range. The evolution of the OPTL, used by the Wearable IoT Device, during the

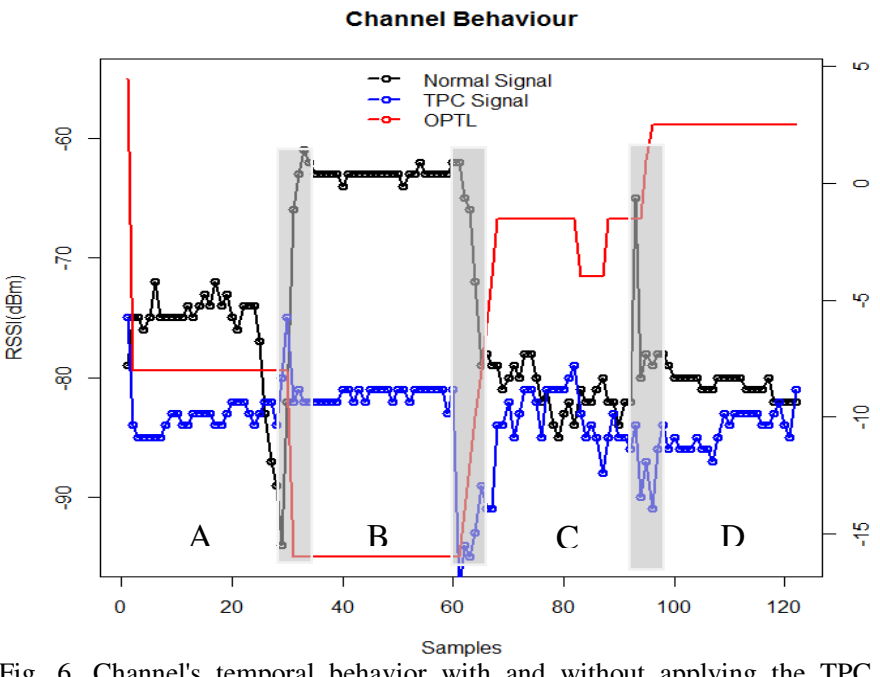

Fig. 6. Channel's temporal behavior with and without applying the TPC mechanism; and OPTL updated over the time by the TPC algorithm. 


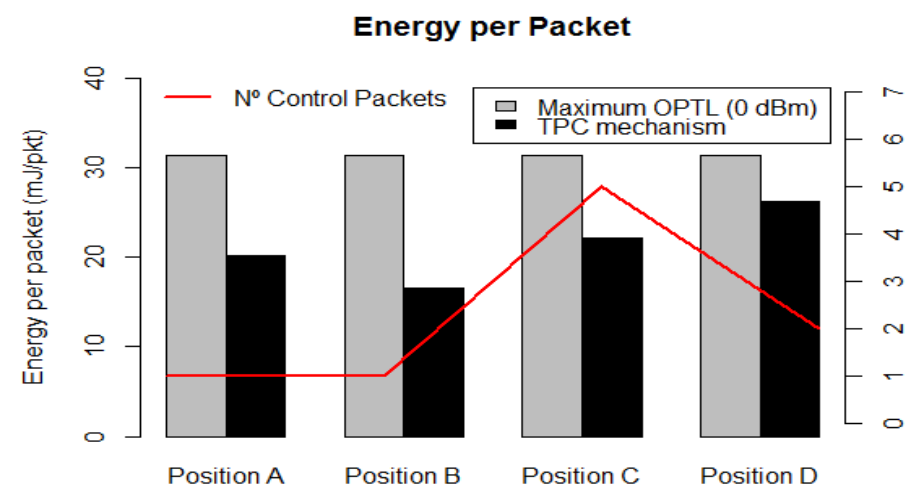

Positions

Fig. 7. Energy Consumption of system with a static OPTL of $\mathrm{dBm}$ and other with the TPC mechanism and number of control packets to reach the OOPT.

transmissions can be seen in the same graph of Fig. 6. Through this results we can conclude that this specific SWS just need a few number of control packets to reach the OOP (one for position $\mathrm{A}$ and $\mathrm{B}$, two for position $\mathrm{D}$ and five for position $\mathrm{C}$ ), and is capable of maintaining within the target RSSI range for long periods of time, independently of the position of infant. Fig. 7 shows the energy consumption for the set of packets transmitted during the practical test, represented in Fig. 6, and the total number of control packets needed to reach the OOPT. The number of control packets sent to reach the OOP is too low. At the present test we checked that it is significantly lower than the number of packets used in the preliminary test, this can be explained by the low number of reflector objects in the surrounding environment. Therefore, we can state that a dynamic OPTL update allow us to reduce significantly the overall energy consumption $(36 \%, 47 \%, 30 \%$ and $4 \%$ for the position $\mathrm{A}, \mathrm{B}, \mathrm{C}, \mathrm{D}$, respectively), with PER of $0 \%$ and low latency. Furthermore, the proposed mechanism allows the reduction of interference with coexisting systems, as the OPTL is usually lower than when used a static value $(0 \mathrm{dBm})$.

\section{CONCLUSIONS}

The SWS is capable of detecting unexpected events and registering several physiological parameters. The energy saving mechanism, optimized for this specific SWS, reduces significantly the energy consumption on wireless communications and radiation level. The proposed TPC mechanism matches features of approaches present in literature. It is based in a closed-loop architecture and the RSSI is adopted to determine the channel state. The accelerometer allows the identification of the position of the infant and, with this information, the system adaptively select the most adequate control (conservative or aggressive). For all the possible positions of the infant, the TPC mechanism revealed to be able to follow the dynamism of the channel, guaranteeing an energy-efficient system - with a maximum of $47 \%$ energy savings - and a reliable communication among devices. Although the mechanism has not been tested in more complex environments (daycare center or hospital), the introduction of a target RSSI margin allow the mechanism to efficiently operate in environments favorable to multipath propagation.

\section{ACKNOWLEDGMENT}

Duarte Fernandes and André G. Ferreira are supported by FCT (grant SFRH/BD/92082/2012 and SFRH/BD/91477/2012 respectively). This work was partially funded by FCT within the Project Scope: Pest-OE/EEI/UI0319/2014, and partially funded by -Programa Operacional Factores de Competitividade - COMPETE and National funds through FCT - Fundação para a Ciência e a Tecnologia- under the project UID/CTM/00264.

\section{REFERENCES}

D. B. Smith, D. Miniutti, T. A. Lamahewa, and L. W. Hanlen, "Propagation Models for Body-Area Networks : A Survey and New Outlook," IEEE Antennas Propag. Mag., vol. 55, no. 5, pp. 97-117, 2013.

[2] S. Movassaghi, M. Abolhasan, J. Lipman, D. Smith, and A Jamalipour, "Wireless Body Area Networks: A Survey," IEEE Commun. Surv. Tutorials, vol. 16, no. 3, pp. 1658-1686, 2014.

[3] D. B. Smith, T. Lamahewa, L. W. Hanlen, and D. Miniutti, "Simple Prediction-Based Power Control for the On-Body Area Communications Channel," in 2011 IEEE International Conference on Communications (ICC), 2011, pp. 1-5.

[4] M. Kim, J. Takada, and S. Member, "Characterization of Wireless On-Body Channel Under Specific Action Scenarios at Sub-GHz Bands," IEEE Trans. Antennas Propag., vol. 60, no. 11, pp. 5364 5372, 2012

[5] K. S. Prabh and J. Hauer, "Opportunistic Packet Scheduling in Body Area Networks," in Proceeding EWSN'11 Proceedings of the 8th European conference on Wireless sensor networks, 2011, pp. 1-16.

[6] M. Quwaider, J. Rao, and S. Biswas, "Body-Posture-Based Dynamic Link Power Control in Wearable Sensor Networks," IEEE Commun. Mag., vol. 48, no. 7, pp. 134-142, 2010.

[7] M. Vallejo, J. Recas, and J. Ayala, "Proactive and Reactive Transmission Power Control for Energy-Efficient On-Body Communications," Sensors, vol. 15, no. 3, pp. 5914-5934, 2015.

[8] W. Lee, B. Lee, and N. Kim, "Hybrid Transmission Power Control for Wireless Body Sensor Systems," Int. J. Distrib. Sens. Networks, vol. 2014 , p. 9,2014

[9] D. Fernades, A. Ferreira, J. Cabral, and J. Mendes, "A Wireless Body Sensor Network based on Dynamic Power Control and Opportunistic Packet Scheduling Mechanisms," in IEEE International Conference on Industrial Technology (ICIT), 2015, pp. $1-6$.

[10] S. Xiao, A. Dhamdhere, V. Sivaraman, and A. Burdett, "Transmission Power Control in Body Area Sensor Networks for Healthcare Monitoring," IEEE J. Sel. Areas Commun., vol. 27, no. 1, pp. 37-48, Jan. 2009.

[11] S. Kim, S. Kim, and D.-S. Eom, "RSSI/LQI-Based Transmission Power Control for Body Area Networks in Healthcare Environment," IEEE J. Biomed. Heal. Informatics, vol. 17, no. 3, pp. 561-571, 2013.

[12] B. Moulton, L. Hanlen, J. Chen, G. Croucher, L. Mahendran, and A. Varis, "Body-Area-Network transmission power control using variable adaptive feedback periodicity," in 2010 Australian Communications Theory Workshop (AusCTW), 2010, pp. 139-144.

[13] M. Quwaider, A. Muhammad, J. Choi, and S. Biswas, "PosturePredictive Power Control in Body Sensor Networks Using LinearQuadratic Gaussian Control," in 2009 First International Conference on Networks \& Communications, 2009, pp. 52-59.

[14] A. G. Ferreira, D. Fernandes, S. Branco, A. P. Catarino, J. L. Monteiro, and J. Cabral, "A Smart Wearable System for Sudden Infant Death for Syndrome Monitoring."

[15] A. ; Y. H. Alomainy, "Radio channel models for UWB body-centric networks with compact planar antenna," in IEEE Antennas and Propagation Society International Symposium 2006, 2006, pp. 2173 $-2176$ 